\title{
Guest Editorial: Special issue on "Application of artificial intelligence in health research"
}

\author{
Siuly Siuly ${ }^{1 *}$ and Xiangliang Zhang ${ }^{2}$
}

\section{Introduction}

Artificial intelligence (AI) is wide-ranging branch of computer science concerned with building smart machines capable of performing tasks the typically require human intelligence. With the rapid advance of technology, AI are becoming increasingly important in almost every realworld impact across various industries, including treatments and healthcare. The applications of AI techniques have sent vast waves across health challenges. AI is being used or trialled for a range of healthcare and research purposes, including detection of disease, management of chronic conditions, delivery of health services, and drug discovery etc.

Modern health treatments are faced with the challenge of acquiring, analysing and applying the large amount of knowledge necessary to solve complex problems. AI in health mainly uses computer techniques to perform clinical diagnoses and suggest treatments. The key objective of this special issue is to present the applications of AI in health research section including the views of leading researchers in the field. This special issue (S.I.) showcases some of the recent developments and novel applications of AI in health fields. This S.I. will provide a forum for the publication of articles that address broad challenges on both theoretical and application aspects of AI in health research. In this issue, we invite scholars and researchers to contribute original research articles that will stimulate the continuing effort on the application of AI approaches to solve health problems.

The open call for this special issue has attracted sixteen papers (16) covering broad range of artificial intelligence technologies and its applications. After two rounds (at

\footnotetext{
*Correspondence: Siuly.Siuly@vu.edu.au

${ }^{1}$ Institute for Sustainable Industries \& Liveable Cities, Victoria University, Melbourne, Australia

Full list of author information is available at the end of the article
}

least) of peer-reviews by a team of international experts, 14 papers were selected to be included in this special issue. Those accepted papers represent a wide spectrum of research under the theme of the special issue. In "Summary of accepted papers" section, we present a brief summation of the selected papers for this special issue and "Conclusion" section provides concluding remarks.

\section{Summary of accepted papers}

This special issue contains state-of-the-art developments in the application of AI in health research, introducing the views of leading researchers in the field. This issue consists of 14 papers that are briefly discussed as follows:

The paper entitled "Ensemble of subspace discriminant classifiers for schistosomal liver fibrosis staging in mice microscopic images" by Ashour et al. [1] introduces a technique to diagnosis parasitic diseases that affect the liver tissues leading to liver fibrosis. This study investigated the microscopic images of the liver tissues at their different stages to assess the fibrosis level for diagnosis and treatment. The effectiveness of the procedure is demonstrated by using numerical simulations.

The article, by Ata et al. [2] "Centroid tracking and velocity measurement of white blood cell in video" presents automated blood cells tracking system to track the blood cell characteristics that indicates several diseases. This paper proposes a green star-based centroid (GSBC) moving white blood cell (WBC) tracking algorithm to measure its velocity and draw its trajectory. The experimental results demonstrated that the proposed system recognized the WBC accurately, while the optical flow identified all other objects lead to its disability to track the WBC.

In "Emotion classification using flexible analytic wavelet transform for electroencephalogram signals" [3], authors proposes a scheme based on flexible analytic wavelet transform (FAWT) for the emotion 
classification. The proposed method used sub-band wise extracted features over the variants of classifier. The weighted $\mathrm{k}$-nearestneighbor $(\mathrm{KNN})$ provides the best emotion classification performance compared to other KNN variants. The proposed single EEG channel based emotion classification method can be used in BCI system for the impaired people to assessed their emotions.

The article, titled "Managing uncertainty in imputing missing symptom value for healthcare of rural India", by Das and Sil [4] proposes a comprehensive framework to impute missing symptom values by managing uncertainty present in the basic health data set (e.g. blood pressure, pulse rate, height-weight, BMI etc.). The proposed method employed fuzzy c-means clustering algorithm to cluster the symptom feature vectors into different disease groups and applied Monte Carlo method to avoid over-fitting problem while imputing missing values of the symptoms.

Elnaggar et al. [5] have contributed an article titled, "An optimized Mamdani FPD controller design of cardiac pacemaker". This article proposes a design of a new cardiac pacemaker based on Mamdani fuzzy proportionalderivative (FPD) controller technique where Mamdani algorithm is the most common algorithm to deal with the human signals. The proposed model is designed, tested, and simulated along with tuning the controller gains using Matlab/Simulink software. The simulation results confirmed the impact of the proposed controller to achieve the optimal HR adaptation to the desired patient's physiological needs at rest.

In article, "Estimation of number of ever born children using zero truncated count model: evidence from Bangladesh Demographic and Health Survey", [6] authors investigated demographic indicators for fertility in Bangladesh considering the reproductive women who had given at least one birth. The paper proposes zero truncated Poisson and zero truncated negative binomial regression models in order to find the best fatted model to estimate number of ever born children using BDHS 2014 dataset.

The article by Liu and Lee [7] titled, "Extracting features with medical sentiment lexicon and position encoding for drug reviews" explores medical sentiment analysis for clinical documents. In this paper, Authors introduce a new feature extraction approach utilising position embeddings to generate a medical domain enhanced sentiment lexicon with position encoding representation for drug review sentiment analysis.

McNabb et al. [8] have contributed an article titled, "Optimizing spatial healthcare assets with Internet of Things". The article proposes the use of Internet of Things (IoT) technology to assist in the optimization of high value clinical spaces and presents phase one of the project where a trial was held in a non-clinical location to evaluate sensor performance. This article gives a greater resolution of automated IoT data to get better optimization decisions of existing high-value spaces and reduce the pressure to expand the footprint of healthcare services and supporting infrastructure.

The article, titled "Features based on variational mode decomposition for identification of neuromuscular disorder using EMG signals" by Nagineni et al. [9] presents an impressive idea based on variational mode decomposition (VMD) method for recognition of neuromuscular disorder from EMG signals. The research findings demonstrate that the proposed method can be beneficial for the diagnosis of neuromuscular disease in clinical application.

In this article, "Similar query was answered earlier: processing of patient authored text for retrieving relevant contents from health discussion forum" the authors [10] introduces an intelligent information retrieval system based on online remedy finders and health-related discussion forums that can help to utilize the repository in various applications. In this paper, authors propose a rule-based approach for the automatic identification of existing similar forum posts given a new post.

Shah et al. [11] titled "Neural networks for mining the associations between diseases and symptoms in clinical notes" explored and evaluated the concept association mining model to reveal the relationships between diseases and symptoms extracted from the clinical notes in the EHR. This concept association mining model is based on word and concept embedding learned through the neural networks. This visualization tool can provide physicians an overview of the medical history of a patient and support decision making.

The paper entitled "Computer assisted system for precise lung surgery based on medical image computing and mixed reality" by Tan et al. [12] introduces a computer surgery system based on MR is designed and implemented for precise lung surgery. The regional growth algorithm is designed to segment lung trachea and lung vessels. This research findings will assist the surgeons to observe and interface precisely the real three-dimensional lung structure of the patient to help them operate accurately the lung surgery.

In "Words prediction based on N-gram model for freetext entry in electronic health records" Yazdani et al. [13] developed a system accelerating the free text entry into electronic health records (EHR) based on the trigram language model. The designed system will reduce the time of typing free text which might be an approach for EHRs improvement in terms of documentation. 
The article by Zhang et al. [14] "Multi-level medical periodic patterns from human movement behaviors" investigates a periodic pattern mining framework for periodic medical pattern detection. Authors utilised two recent spatio-temporal (ST) hierarchical periodic pattern mining approaches to find hierarchical medical periodic patterns, and demonstrate the usability and applicability of the proposed framework in medical settings using a popular real-world trajectory dataset. This proposed method will be useful for the discovery and diagnosis of diseases for patients.

\section{Conclusions}

As mentioned before, this S.I. selected 14 papers, which illustrate the diverse range of issues of application of AI in the health area. The papers included in this special issue are representatives of the current research challenges in advanced understanding, modeling and implementation of AI in health filed which are capable of solving a variety of clinical and health problems. It is expected that these papers can provide researchers with valuable resources and motivations to work on the challenging issues in this research theme. We would like to thank the Editor-in-Chief of the journal, Professor Yanchun Zhang for his huge support for this issue. Our special thanks go to all editorial staffs, especially Melissa Fearon, Keerthana Tananjayan and Katrina Turner for their valuable and prompt support throughout the preparation and publication of this special issue. We would like to thank all authors for their contributions to this special issue. We also extend our thanks to all reviewers for their hard work to ensure the high quality of accepted papers.

\section{Author details}

${ }^{1}$ Institute for Sustainable Industries \& Liveable Cities, Victoria University, Melbourne, Australia. ${ }^{2}$ King Abdullah University of Science and Technology, Thuwal, Saudi Arabia.
References

1. Ashour AS, Guo Y, Hawas AR, Xu G. Ensemble of subspace discriminant classifiers for schistosomal liver fibrosis staging in mice microscopic images. Health Inf Sci Syst. 2018;6:21. https://doi.org/10.1007/s1375 5-018-0059-8.

2. Ata MM, Ashour AS, Guo Y, Abd Elnaby MM. Centroid tracking and velocity measurement of white blood cell in video Mohamed Mahe. Health Inf Sci Syst. 2018;6:20. https://doi.org/10.1007/s13755-018-0060-2.

3. Bajaj V, Taran S, Sengur A. Emotion classifcation using fexible analytic wavelet transform for electroencephalogram signals. Health Inf Sci Syst. 2018;6:12. https://doi.org/10.1007/s13755-018-0048-y.

4. Das S, Sil J. Managing uncertainty in imputing missing symptom value for healthcare of rural India. Health Inf Sci Syst. 2019;7:5. https://doi. org/10.1007/s13755-019-0066-4.

5. Elnaggar MI, Ashour AS, Guo Y, El-Khobby HA, Abd Elnaby MM. An optimized Mamdani FPD controller design of cardiac pacemaker. Health Inf Sci Syst. 2019;7:2. https://doi.org/10.1007/s13755-018-0063-z.

6. Kiser $\mathrm{H}$, Hossain Md A. Estimation of number of ever born children using zero truncated count model: evidence from Bangladesh Demographic and Health Survey. Health Inf Sci Syst. 2019;7:3. https://doi.org/10.1007/ s13755-018-0064-y.

7. Liu S, Lee I. Extracting features with medical sentiment lexicon and position encoding for drug reviews. Health Inf Sci Syst. 2019;7:11. https://doi. org/10.1007/s13755-019-0072-6.

8. McNabb T, Myers T, Wicking K, Lei L, Xiang W. Optimizing spatial healthcare assets with Internet of Things. Health Inf Sci Syst. 2018;6:11. https:// doi.org/10.1007/s13755-018-0052-2.

9. Nagineni S, Taran S, Bajaj V. Features based on variational mode decomposition for identification of neuromuscular disorder using EMG signals. Health Inf Sci Syst. 2018;6:13. https://doi.org/10.1007/s13755-018-0050-4.

10. Saha SK, Prakash A, Majumder M. "Similar query was answered earlier": processing of patient authored text for retrieving relevant contents from health discussion forum. Health Inf Sci Syst. 2019;7:4. https://doi. org/10.1007/s13755-019-0067-3.

11. Shah S, Luo X, Kanakasabai S, Tuason R, Klopper G. Neural networks for mining the associations between diseases and symptoms in clinical notes. Health Inf Sci Syst. 2019;7:1. https://doi.org/10.1007/s1375 5-018-0062-0.

12. Tan W, Ge W, Hang Y, Simeng W, Liu S, Liu M. Computer assisted system for precise lung surgery based on medical image computing and mixed reality. Health Inf Sci Syst. 2018;6:10. https://doi.org/10.1007/s1375 5-018-0053-1.

13. Yazdani A, Safdari R, Golkar A, Niakan Kalhori SR. Words prediction based on $\mathrm{N}$-gram model for free-text entry in electronic health records. Health Inf Sci Syst. 2019;7:6. https://doi.org/10.1007/s13755-019-0065-5.

14. Zhang D, Lee K, Lee I. Multi-level medical periodic patterns from human movement behaviors. Health Inf Sci Syst. 2019;7:9. https://doi. org/10.1007/s13755-019-0070-8.

Publisher's Note Springer Nature remains neutral with regard to jurisdictional claims in published maps and institutional affiliations. 